\title{
Analysis of Boundary Layer Flow and Heat Transfer for two Classes of Viscoelastic Fluid Over a Stretching Sheet with Heat Generation or Absorption
}

\author{
K. Bhattacharyya ${ }^{a}$, M. S. Uddin ${ }^{b *}$, G. C. Layek ${ }^{a}$ and W. Ali Pk. \\ ${ }^{a}$ Department of Mathematics, The University of Burdwan, Burdwan-713104, West Bengal, India \\ ${ }^{b}$ Mathematics Discipline, Khulna University, Khulna-9208 and ${ }^{c}$ Department of Mathematics, \\ University of Rajshahi, Rajshahi-6205, Bangladesh
}

\begin{abstract}
In this paper, we obtained solutions of boundary layer flow and heat transfer for two classes of viscoelastic fluid over a stretching sheet with internal heat generation or absorption. In the analysis, we consider second-grade fluid and Walter's liquid B. The governing equations are transformed into self-similar ordinary differential equations by similarity transformations. The flow equation relating to momentum is solved analytically and then the heat equation using the Kummer's function. The analysis reveals that for the increase in magnitude of viscoelastic parameter both the velocity and temperature for a fixed point increase for second-grade fluid and both decrease for Walter's liquid B. Due to increase in Prandtl number and heat sink parameter, the thermal boundary layer thickness reduces, whereas increasing heat source parameter increases that thickness.
\end{abstract}

Key words: Boundary layer flow, Heat transfer, Viscoelastic fluid, Stretching sheet, Heat generation or absorption

\section{Introduction}

Flow induced by a stretching sheet of Newtonian or nonNewtonian fluid is a classical problem in fluid mechanics. Study of momentum and heat transfer in laminar boundary layer flow of a viscoelastic fluid over a linearly stretching sheet is of great importance in polymer processing industries e.g., in manufacturing artificial fibers and artificial films etc. The concept of viscous flow due to a linearly stretching sheet was introduced by Crane (1970) and obtained an closed form analytical solution. The pioneering work of Crane was extended by Rajagopal et al. (1984) by taking viscoelastic fluid and also Siddappa and Abel (1985) discussed some other important aspects of flow of non-Newtonian fluid over stretching sheet. Troy et al. (1987) established the uniqueness of solution of the flow of second-grade fluid over a stretching sheet. But two years latter, Chang (1989) showed that the solution of the flow of viscoelastic fluid is not unique and he obtained the different forms of non-unique solutions. In 1987, Bujurke et al. presented the heat transfer analysis for the flow of second order viscoelastic fluid over a stretching sheet. Lawrence and Rao (1992) also demonstrated the heat transfer in the flow of viscoelastic fluid past a stretching sheet. Andersson (1992) showed the magnetic effect on the flow of Walter's liquid B over a stretching sheet.
Cortell (1994) obtained the similarity solution for the flow and heat transfer of a viscoelastic fluid over a stretching sheet.

In case of heat transfer analysis, the thermal radiation and internal heat generation or absorption are very important physically. Siddheshwar and Mahabaleswar (2005) investigated the radiation effects on MHD flow of a viscoelastic fluid and heat transfer over a stretching sheet taking into account the internal heat generation/absorption. Khan (2006) studied the effects of radiation as well as heat source/sink and mass suction/blowing on heat transfer in viscoelastic fluid flow over a stretching surface. Cortell (2007) analyzed the mass transfer with chemically reactive species for two classes of viscoelastic fluid viz. second-grade fluid and Walter's liquid B over a porous stretching sheet.

In our study, we investigate the effects of heat generation or absorption on the flow of two different classes of viscoelastic fluid namely, second-grade and Walter's liquid B over a stretching sheet which is being stretched linearly. We obtained the exact solutions for both momentum and heat equations. The solution of self-similar heat conducting equa-

*Corresponding author: E-mail: sharif_ku@yahoo.com 
tion is obtained in the form of Kummer's function (a confluent hypergeometric function). The numerical solutions are also obtained using shooting method. Actually, this work is the generalization of the study of Cortell (1994).

\section{Formulation of the flow and heat transfer problems}

We consider a steady laminar flow of an incompressible viscoelastic fluid over a plane sheet coinciding with the plane $y=0$, the flow being confined in the plane $y>0$. The motion is caused due a linear stretching of the sheet because of simultaneous application of two equal opposite forces along the $x$ axis so that the sheet stretched keeping the origin fixed and the physical sketch of the flow dynamics is given in Fig. 1. The boundary layer equations for momentum and heat transfer may be written as follows:

$$
\frac{\partial u}{\partial x}+\frac{\partial v}{\partial y}=0
$$

$u \frac{\partial u}{\partial x}+v \frac{\partial u}{\partial y}=v \frac{\partial^{2} u}{\partial y^{2}} \pm k_{0}$

$\left\{u \frac{\partial^{3} u}{\partial x \partial y^{2}}+\frac{\partial u}{\partial x} \frac{\partial^{2} u}{\partial y^{2}}-\frac{\partial u}{\partial y} \frac{\partial^{2} u}{\partial x \partial y}+v \frac{\partial^{3} u}{\partial y^{3}}\right\}$

and $\quad u \frac{\partial T}{\partial x}+v \frac{\partial T}{\partial y}=\frac{\kappa}{\rho c_{p}} \frac{\partial^{2} T}{\partial y^{2}}+\frac{Q_{0}}{\rho c_{p}}\left(T-T_{\infty}\right)$

where $u$ and $v$ are velocity components in $x$ and $y$ directions respectively, $v(=\mu / \rho)$ the kinematic fluid viscosity, $\rho$ is the fluid density, $\mu$ is the coefficient of fluid viscosity, $k_{0}$ is coefficient of viscoelasticity, $T$ is the temperature, $k$ is the fluid thermal conductivity, $c_{p}$ is the specific heat, $Q_{0}$ is the heat generation or absorption coefficient, $T_{o o}$ is the free stream temperature. The positive sign in the right hand side of equation (2) is corresponding to second-grade fluid (Cortell, 1994 and 2006) whereas the negative sign is for Walter's liquid B (Prasad et al., 2000 and Khan et al., 2003) also termed as second-order fluid (Khan and Sanjayanand, 2000).

The appropriate boundary conditions for the velocity components and the temperature are given by

$u=c x, v=0$ at $y=0 ; u \rightarrow 0, \partial u / \partial y \rightarrow 0$ as $y \rightarrow \infty$

and , $T=T_{w}$ at $y=0 ; T \rightarrow T_{\infty}$ as $y \rightarrow \infty$

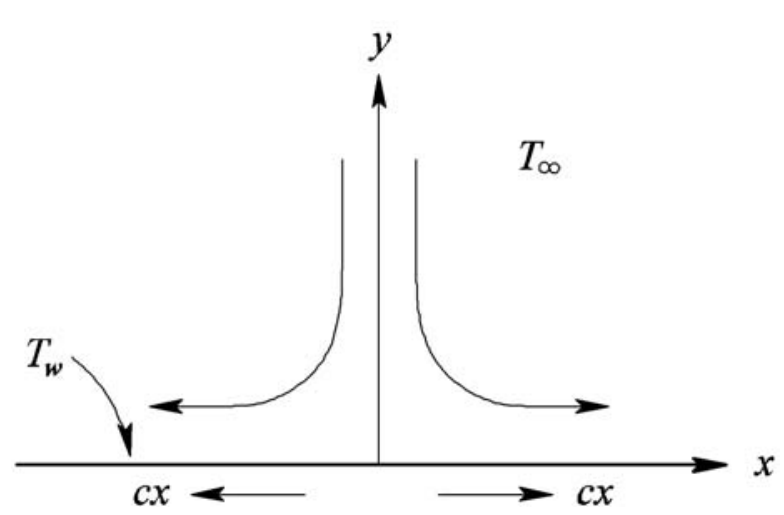

Fig. 1: Physical sketch of the flow

where $c$ is stretching constant with $c>0, T_{\mathrm{w}}$ temperature of the sheet assumed to be constant. The last condition of (4) is the augmented condition because the flow is in an unbounded domain, which had been discussed by Garg and Rajagopal (1992).

We now introduce the stream function $\psi(x, y)$ as

$$
u=\partial \psi / \partial y \text { and } v=-\partial \psi / \partial x
$$

The mass-conservation equation (1) is satisfied automatically and the momentum equation (2) and temperature equation (3) take the following forms.

$$
\begin{aligned}
& \frac{\partial \psi}{\partial y} \frac{\partial^{2} \psi}{\partial x \partial y}-\frac{\partial \psi}{\partial x} \frac{\partial^{2} \psi}{\partial y^{2}}=v \frac{\partial^{3} \psi}{\partial y^{3}} \pm \\
& k_{0}\left\{\frac{\partial \psi}{\partial y} \frac{\partial^{4} \psi}{\partial x \partial y^{3}}+\frac{\partial^{2} \psi}{\partial x \partial y} \frac{\partial^{3} \psi}{\partial y^{3}}-\frac{\partial^{2} \psi}{\partial y^{2}} \frac{\partial^{3} \psi}{\partial x \partial y^{2}}-\frac{\partial \psi}{\partial x} \frac{\partial^{4} \psi}{\partial y^{4}}\right\} \\
& \text { and } \frac{\partial \psi}{\partial y} \frac{\partial T}{\partial x}-\frac{\partial \psi}{\partial x} \frac{\partial T}{\partial y}=\frac{\kappa}{\rho c_{p}} \frac{\partial^{2} T}{\partial y^{2}}+\frac{Q_{0}}{\rho c_{p}}\left(T-T_{\infty}\right)
\end{aligned}
$$

Accordingly, the boundary conditions in (4) reduce to

$$
\frac{\partial \psi}{\partial y}=c x, \frac{\partial \psi}{\partial x}=0 \text { at } y=0 ; \frac{\partial \psi}{\partial y} \rightarrow 0, \frac{\partial^{2} \psi}{\partial y^{2}} \rightarrow 0 \text { as } y \rightarrow \infty
$$

Next, we introduce the dimensionless variables for $\psi$ and $T$ as given below:

$\psi=\sqrt{c v} x f(\eta)$ and $T=T_{\infty}+\left(T_{w}-T_{\infty}\right) \theta(\eta)$

The similarity variable denoted by $\eta$ is given by $\eta=y(\mathrm{c} / v))^{1 / 2}$. 
Using the dimensionless variables and similarity variable, the above equations finally take the self-similar forms given below:

$$
\left(f^{\prime}\right)^{2}-f f^{\prime \prime}=f^{\prime \prime \prime}+\lambda\left[2 f f^{\prime \prime \prime}-\left(f^{\prime \prime}\right)^{2}-f f^{i v}\right]
$$

and $\theta^{\prime \prime}+\operatorname{Pr}\left[f \theta^{\prime}-\delta \theta\right]=0$

where $\lambda= \pm k_{0} c / v$ is the viscoelastic parameter with $\lambda>0$ corresponding to the second-grade fluid and $\lambda<0$ for the Walter's liquid $\mathrm{B}, P r=\mu c_{\mathrm{p}} / k$ is the Prandtl number and $\delta=Q_{0} / \rho c_{p} c$ is the heat source $(\delta<0)$ or $\operatorname{sink}(\delta>0)$ parameter.

The boundary conditions (7) and (5) also transform to

$f(\eta)=0, f^{\prime}(\eta)=1$ at $\eta=0 ; f^{\prime}(\eta) \rightarrow 0, f^{\prime \prime}(\eta)$

$\rightarrow 0$ as $\eta \rightarrow \infty$

and $\quad \theta(\eta)=1$ at $\eta=0 ; \theta(\eta) \rightarrow 0$ as $\eta \rightarrow \infty$

\section{Solutions of self-similar equations}

The self-similar equation (9) with the boundary condition (11) has a solution of the form

$$
\begin{aligned}
& f(\eta)=\frac{1}{\alpha}(1-\exp (-\alpha \eta)) \\
& \alpha=\sqrt{1 /(1+\lambda)} .
\end{aligned}
$$

According the sign of $\lambda$, we can consider the fluid to be either second-grade $(\lambda>0)$ or Walter's liquid $\mathrm{B}(\lambda<0,|\lambda|<1)$. Also, replacing $\lambda$ by $-\lambda$ in the equations (13) and (14) earlier analyses of Cortell (1994) and Khan (in nonporous medium) (2006) can be obtained.

The analytic solution of equation (10) with respective boundary condition (12) can be written in form of hypergeometric function:

$\theta(\eta)=\frac{e^{-\alpha(G+H) \eta} M\left(G+H, 1+2 H,-\left(\operatorname{Pr} / \alpha^{2}\right) e^{-\alpha \eta}\right)}{M\left(G+H, 1+2 H,-\operatorname{Pr} / \alpha^{2}\right)}$

Where $G=\operatorname{Pr} / 2 \alpha^{2}$ and $H=\left(P r^{2}-4 \delta \alpha^{2} P r\right)^{1 / 2} / 2 \alpha^{2}$

The confluent hypergeomertic function $\mathrm{M}$ is the Kummer's function (Abramowitz and Stegun, 1965) and is defined by

$M\left(g_{0}, h_{0}, z\right)=1+\sum_{n=1}^{\infty} \frac{\left(g_{0}\right)_{n} z^{n}}{\left(h_{0}\right)_{n} n !}$

$$
\begin{aligned}
& \text { with }\left(g_{0}\right)_{n}=g_{0}\left(g_{0}+1\right)\left(g_{0}+2\right) \mathbf{L} \mathbf{L}\left(g_{0}+n-1\right) \\
& \text { and }\left(h_{0}\right)_{n}=h_{0}\left(h_{0}+1\right)\left(h_{0}+2\right) \mathbf{L} \mathbf{L}\left(h_{0}+n-1\right) .
\end{aligned}
$$

\section{Numerical solutions}

The nonlinear coupled differential equations (9) and (10) along with the boundary conditions form a boundary value problem (BVP) and is solved using shooting method, by converting it into an initial value problem (IVP). In this method we have to choose a suitable finite value of $\eta \rightarrow_{\circ}$, say $\eta_{o}$, We set following first-order systems

$$
\begin{aligned}
& f^{\prime}=p, p^{\prime}=q, q^{\prime}=r, r^{\prime}=\left[r+\lambda\left(2 p r-q^{2}\right)-p^{2}\right. \\
& +f q] /(\lambda f) \\
& \text { and } \theta^{\prime}=z, z^{\prime}=-\operatorname{Pr}[f z-\delta \theta]
\end{aligned}
$$

with the boundary conditions

$$
f(0)=0, p(0)=1, q(0)=0, \theta(0)=1 \text {. }
$$

To solve (17) and (18) as an IVP we must need values for $r(0)$ i.e. $f^{\prime \prime \prime}(0)$ and $z(0)$ i.e. $\theta^{\prime}(0)$ but no such values are given. The initial guess values for $f^{\prime \prime \prime}(0)$ and $\theta^{\prime}(0)$ are chosen and applying fourth order Runge-Kutta method a solution is obtained. Then we compare the calculated values of $f^{\prime}(\eta)$ and $\theta(\eta)$ at $\eta_{\infty}(=20)$ with the given boundary conditions $f\left(\eta_{\infty}\right)=0$ and $\theta\left(\eta_{\infty}\right)=0$ and adjust values of $f^{\prime \prime \prime}(0)$ and $\theta^{\prime}(0)$ using Secant method to give better approximation for the solution. The step-size is taken as $\Delta \eta=0.01$. This process is repeated until we get the results correct up to the desired accuracy of $10^{-6}$ level. The comparisons of numerical and exact solutions are presented in Figs. 2 and 3 and it is observed that the numerical solutions are matched well with the exact solutions. On the other hand, the Secant method regarding the selection of shoot values is convergent, which is already proved.

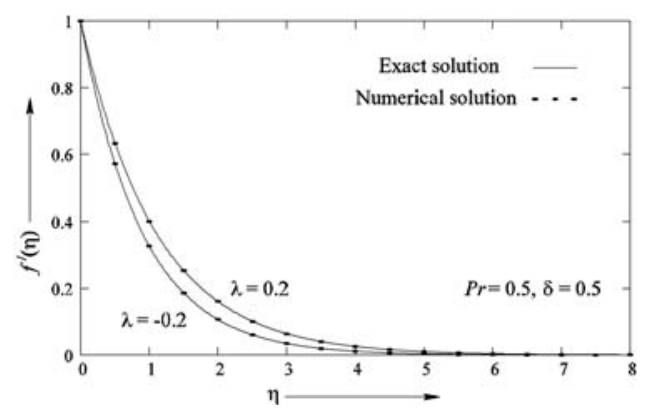

Fig. 2: Comparison of velocity profiles 


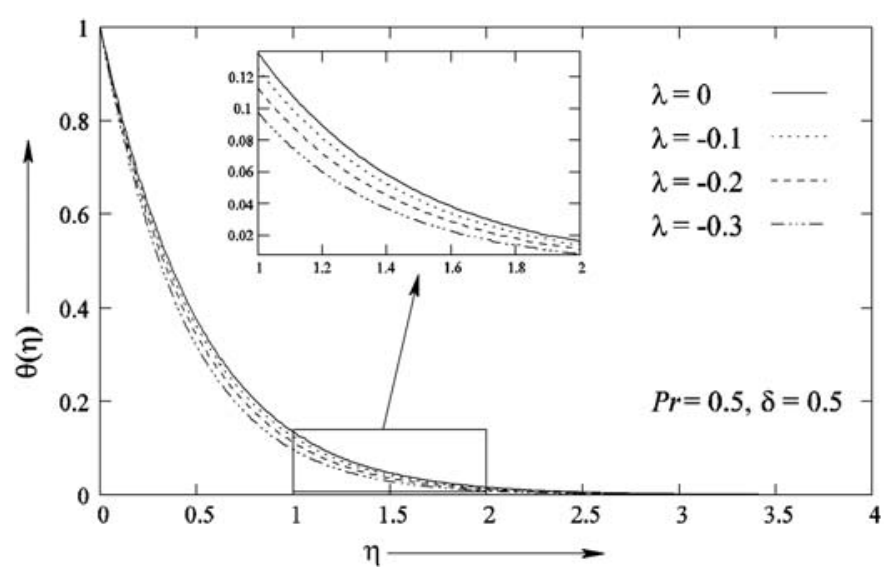

Fig. 3: Comparison of temperature profiles

\section{Results and Discussions}

The exact solutions for different values of parameters are plotted in figures and the physical characteristics are given.

The solution curves for velocity are plotted and are shown in Figs $4 \mathrm{a}$ and $4 \mathrm{~b}$ for several value of $\lambda$ for second-grade $(\lambda>0)$ and Walter's liquid $\mathrm{B}(\lambda<0)$ respectively. It is noticed that the dimensionless velocity represented by $f^{\prime}(\eta)$ increases with an increase in $\lambda$ with $\lambda>0$ i.e. for second order fluid and on the other hand, it decreases as magnitude of $\lambda$ increases when $\lambda<0$ i.e. in case of Walter's liquid B. So, two opposite behaviours in velocity curves for two different classes of viscoelastic fluid are noticed.

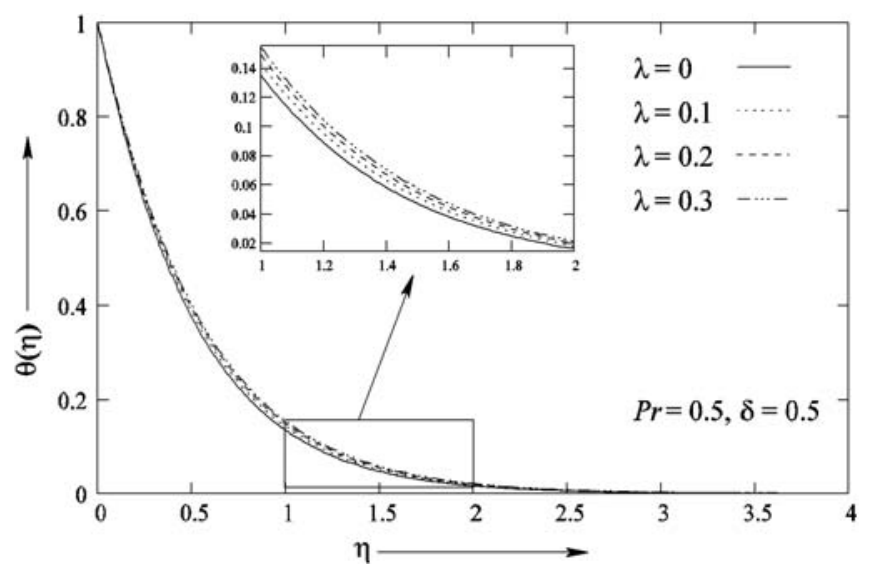

Fig. 4a: Velocity profiles for several values of $\lambda$ for second-grade fluid (i.e. $\lambda>0$ )

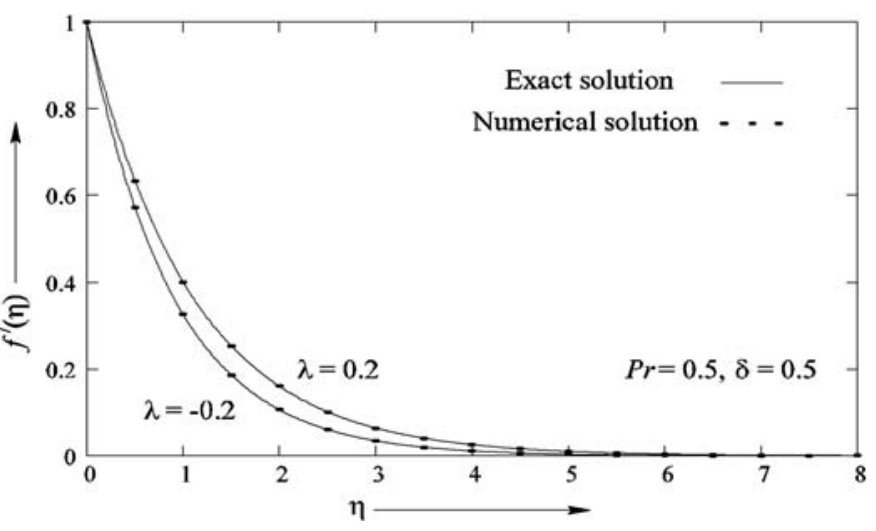

Fig. 4b: Velocity profiles for several values of $\lambda$ Walter's liquid B (i.e. $\lambda<0$ )

Now the numerical solution curves for the temperature distribution for several values of $\lambda$ are presented in Figs $5 \mathrm{a}$ and 5b. In Fig 5a, for the second-grade fluid the dimensionless temperature $\theta(\eta)$ increases with increasing $\lambda$, whereas from Fig5b i.e. for the Walter's liquid B $\theta(\eta)$ decreases when the

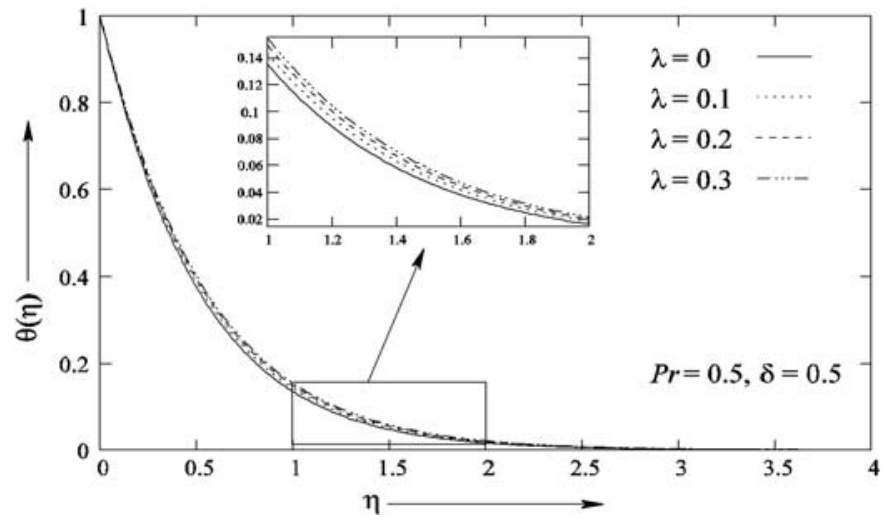

Fig. 5a: Temperature profiles for several values of $\lambda$ for second-grade fluid (i.e. $\lambda>0$ )

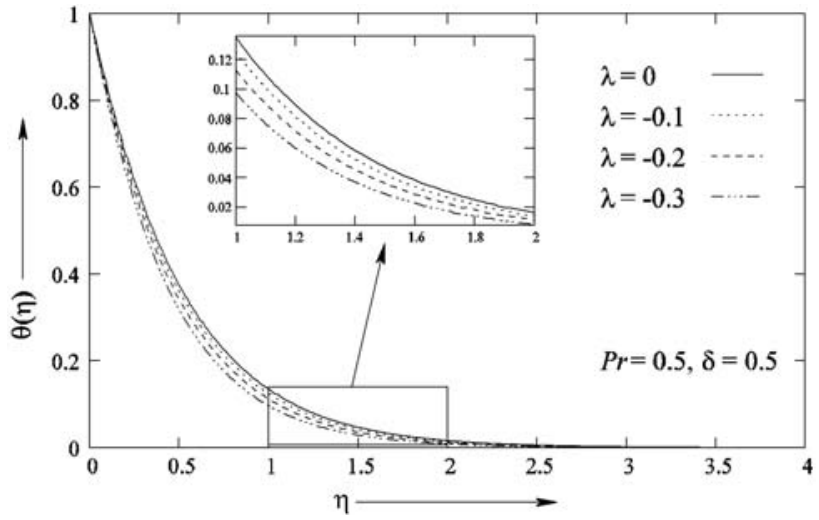

Fig. 5b: Temperature profiles for several values of $\lambda$ Walter's liquid B (i.e. $\lambda<0$ ) 
magnitude of $\lambda$ increases. The natures of the dimensionless temperature profiles are same as that of the dimensionless velocity profiles.

The deviation in the temperature profiles for the variation of the Prandtl number is demonstrated in Figs $6 \mathrm{a}$ and $6 \mathrm{~b}$. For both type of viscoelastic fluids, the temperature as well as the thermal boundary layer thickness decreases rapidly with increasing values of $P r$. Thus the Prandtl number affects the temperature distribution for both types of fluids in similar manner.

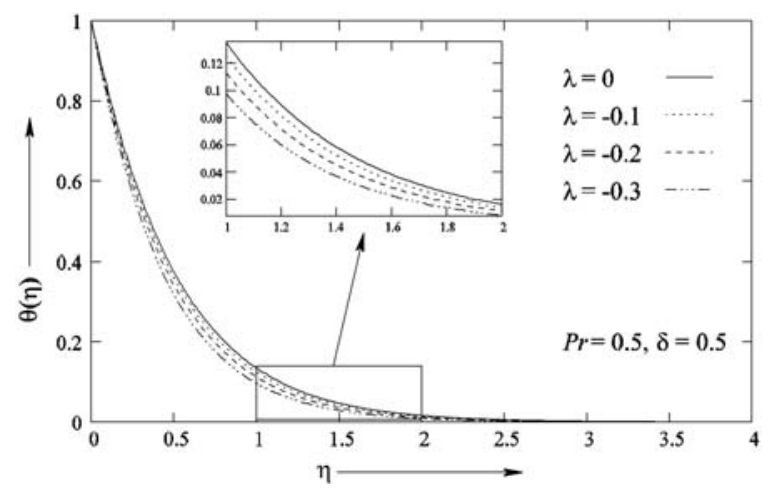

Fig. 6a: Temperature profiles for several values of $P r$ for second-grade fluid (i.e. $\lambda>0$ )

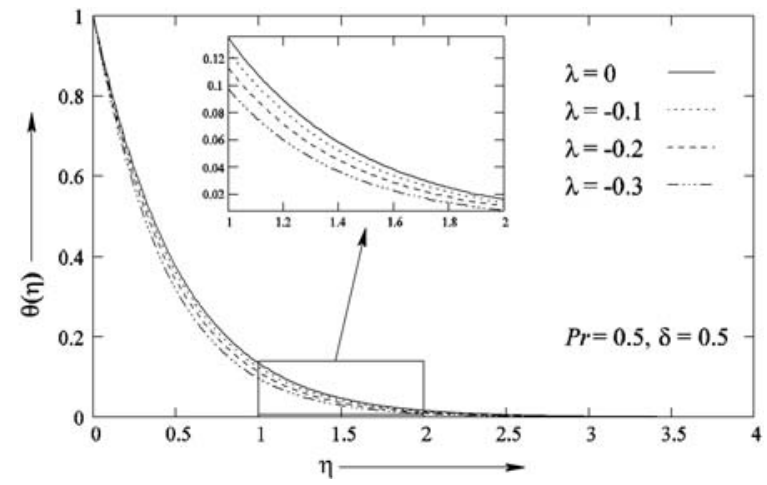

Fig. 6b: Temperature profiles for several values of $\boldsymbol{P r}$ for Walter's liquid B (i.e. $\lambda<0$ )

Finally, the effects of internal heat source or sink on the heat transfer are exhibited in the Figs7a and $7 \mathrm{~b}$. Due to the increase in the heat source or sink parameter the thickness of the thermal boundary layer is reduced and the temperature at a point decreases. Thus, with the increase in strength of heat source the temperature and the thermal boundary layer thickness increase but, the outcome of increase in strength of the heat sink is totally opposite i.e. the temperature and the thermal boundary layer thickness decrease with increase in sink strength. The above thermal behaviours are found in both second-grade fluid and Walter's liquid B.

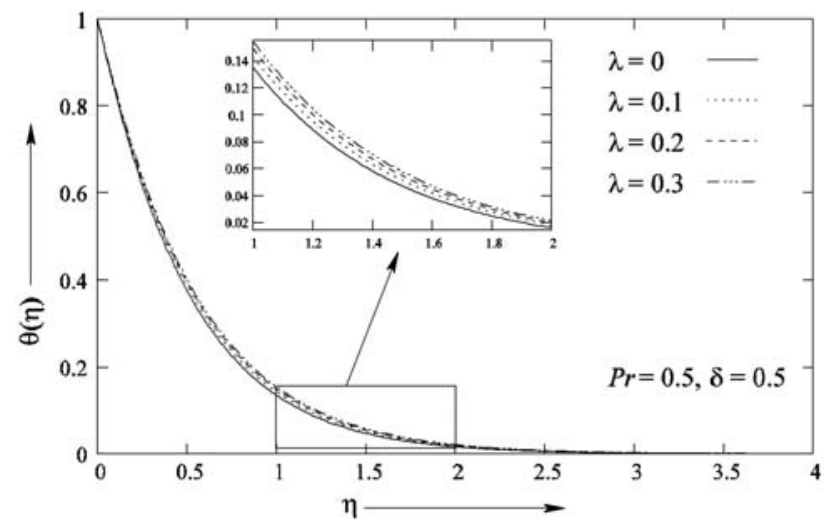

Fig. 7a: Temperature profiles for several values of $\delta$ for second-grade fluid (i.e. $\lambda>0$ )

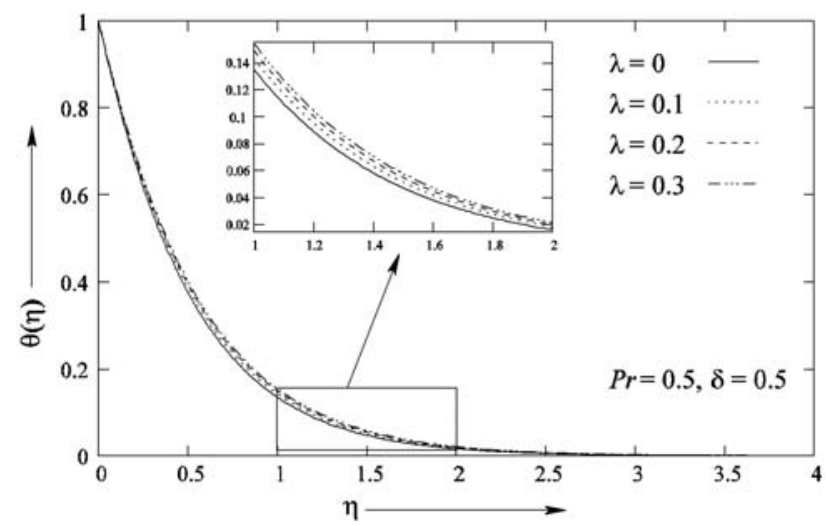

Fig. 7b: Temperature profiles for several values of $\delta$ for Walter's liquid B (i.e. $\lambda<0$ )

\section{Conclusion}

The Objective of our study is to investigate the flow behaviours and heat transfer for two classes of viscoelastic fluid over a stretching sheet with internal heat generation or absorption. Using similarity variables, the momentum and heat equations are transferred into self-similar ordinary differential equations. The momentum equation is solved analytically and also the heat equation using a confluent hypergeomertic function, the well known Kummer's function. The numerical solutions are also obtained using shooting method and the exact and numerical solutions are well matched. The exact solutions are presented in some figures and the following conclusions can be drawn: 
1. Due to increase in magnitude of viscoelastic parameter the velocity and temperature increase for second-grade fluid and decrease for Walter's liquid B.

2. For both fluids, increase in Prandtl number reduces the thermal boundary layer thickness.

3. The temperature at a point increases with the heat source strength and decreases with heat sink strength for second-grade as well as Walter's B fluids.

\section{Acknowledgement}

One of the authors (K.B.) gratefully acknowledges the financial support of National Board for Higher Mathematics (NBHM), DAE, Mumbai, India for pursuing this work. Also, authors are thankful to the honourable reviewer for his/her valuable comments and suggestions.

\section{References}

Abramowitz M. and Stegun F. (1965). Handbook of Mathematical Functions, Dover, New York.

Andersson H. I. (1992). Note: MHD flow of a viscoelastic fluid past a stretching surface. Acta Mech., 95: 227230 .

Bujurke N. M., Biradar S. N. and Hiremath P. S. (1987). Second order fluid flow past a stretching sheet with heat transfer. ZAMP, 38: 653-657.

Chang W. D. (1989). The non-uniqueness of the flow of a viscoelastic fluid over a stretching sheet. Quart. Appl. Math., 47: 365-366.

Cortell R. (1994). Similarity solutions for flow and heat transfer of a viscoelastic fluid over a stretching sheet. Int. J. Non-Linear Mech., 29: 155-161.

Cortell R. (2006). A note on flow and heat transfer of a viscoelastic fluid over a stretching sheet. Int. J. NonLinear Mech., 41: 78-85.

Cortell R. (2007). Toward an understanding of the motion and mass transfer with chemically reactive species for two classes of viscoelastic fluid over a porous stretching sheet. Chem. Eng. and Process, 46: 982-989.

Crane L. J. (1970). Flow past a stretching plate. ZAMP, 21: 645-647.
Garg V. K. and Rajagopal K. R. (1992). Flow of nonNewtonian fluid past a wadge. Acta Mech., 93: 53-61.

Khan S. K., Abel M. S. and Sonth R. M. (2003). Viscoelastic MHD flow, heat and mass transfer over a porous stretching sheet with dissipation of energy and stress work. Heat Mass trans., 40:47-57.

Khan S. K. and Sanjayanand E. (2005). Viscoelastic boundary layer flow and heat transfer over an exponentially stretching sheet. Int. J. Heat Mass Trans., 48:15341542.

Khan S. K. (2006). Heat transfer in a viscoelastic fluid flow over a stretching surface with heat source/sink, suction/blowing and radiation. Int. J. Heat Mass Trans., 49: 628-639.

Lawrence P. S. and Rao B. N. (1992). Heat transfer in the flow of a viscoelastic fluid over a stretching sheet. Acta Mech., 93: 53-61.

Prasad K. V., Abel M. S. and Khan S. K. (2000). Momentum and heat transfer in visco-elastic fluid flow in a porous medium over a non-isothermal stretching sheet. $J$. Num. Meth. Heat Fluid Flow, 10: 786-801.

Rajagopal K. R., Na T. Y. and Gupta A. S. (1984). Flow of viscoelastic fluid over a stretching sheet. Rheol Acta, 23: 213-215.

Siddappa B. and Abel M. S. (1985). Non-Newtonian flow past a stretching plate. ZAMP, 36: 890-892.

Siddheshwar P. G. and Mahabaleswar U. S. (2005) Effects of radiation and heat source on MHD flow of a viscoelastic liquid and heat transfer over a stretching sheet. Int. J. Non-Linear Mech., 40: 807-820.

Troy W. C., E. A. Overman E. A., Ermentrout H. G. B. and Keerner J. P. (1987). Uniqueness of the flow of a second order fluid past a stretching sheet. Quart. Appl. Math., 44: 753-755.

Received: May 18, 2010;

Accepted : January 27, 2011 